95\% CI: 2.0 to 4.5 ) were more likely to have paid work compared to those without chronic disease in 2002.

Conclusion The present study showed that people with a chronic disease aged 55-64 years were less likely to be involved in paid work, but we did not find indications for differences in participation in providing informal care or volunteer work. However, we did find a time effect for participation in paid work for people with and without a chronic disease in 2012 compared to 2002. Future research should focus on differences in social participation within the heterogeneous group with chronic disease, as differences may be present in subgroups.

\section{PROLONGING WORKING LIFE BY ENGAGING EMPLOYEES IN DEVELOPING WORK WELLBEING WITH TOTAL OPENNESS AND TRANSPARENCY WITH THE KIVAQ METHOD}

${ }^{1}$ Näsman Ove ${ }^{*},{ }^{2}$ Strandberg Erika. ${ }^{1} M D$, KivaQ Ltd, Kimitoön, Finland; ${ }^{2}$ MSSc, Municipality of Kimitoön, Kimitoön, Finland

\subsection{6/oemed-2018-ICOHabstracts. 124}

Introduction Employee wellbeing correlates with long working life. Every organisation should have a structured program for developing work wellbeing. The KivaQ method has been used in Finland in many successful projects.

The KivaQ method The KivaQ method consists of a questionnaire and a development workshop. The online questionnaire is fast, easy to use, validated, affordable and engaging. The seven standard questions are in 17 languages. All information is accessible at the website www.kivaq.fi including the questions and reference materials from different industries in the analysing module. The structured workshop has seven ppt-bottoms for the facilitator. A KivaQ method guide is available in English and Finnish. In 2016 a small municipality in Finland with 584 employees executed the KivaQ questionnaire with total openness. Every employee got the password and could analyse the results of the survey. In 2017 the survey was repeated with total openness. The openness was studied by constructing two additional questions in the 2017 survey.

Result In the 2017 survey 27,6\% of the respondents reported, that they had used the possibility and themselves analysed the survey in 2016. Of the respondents $69,2 \%$ thought that the new way with openness in the personnel survey was good or very good. Only $1,5 \%$ thought the idea was bad or very bad. $29,3 \%$ had no opinion.

Discussion The results indicate that increased organisational openness also in surveys is welcomed by the majority of the personnel. This may increase the informal discussions on work wellbeing and result in increased investment in wellbeing. Some teams in the example municipality are already discussing participating in the KivaQ workshop. This may lead to prolonged working life. The authors call for more testing with total openness in surveys.

\section{EARLY FUNCTIONAL AGEING AS DETERMINANT FOR JOB INTERRUPTION: A 4-YEAR FOLLOW UP}

${ }^{1} \mathrm{FM}$ Fischer, ${ }^{2} \mathrm{MC}$ Martinez. ${ }^{1}$ Department of Environmental Health, School of Public Health, University of São Paulo, Brazil; ${ }^{2}$ WAF Informática, São Paulo, Brazil

10.1136/oemed-2018-ICOHabstracts. 125
Introduction Functional ageing is a progressive work ability impairment. It is associated with negative outcomes including early retirement, unemployment and leaving the profession. The aim of this study is to investigate whether early functional ageing plays a determinant role in job interruption in the short-to-medium run among hospital employees.

Methods A 4 year cohort (2008-2012) of 1060 hospital workers in São Paulo, Brazil. At baseline participants filled a questionnaire about demographic, occupational and lifestyle items, and the Brazilian version of Job Stress Scale (JSS) and Work Ability Index (WAI). The outcome variable 'job interruption at the end of follow up' was no (active employees)and yes (transient sick leave and job termination). This information was obtained from human resources department. Associations between functional ageing and other independent variables with job interruption were identified by multiple logistic regression.

Results Participants' average age at baseline were 32.5 y $(\mathrm{SD}=8.5), 29.4 \%$ were over 40 , and $69.8 \%$ were females. Relative to psychosocial work factors, $67.8 \%$ corresponded to active work, $11.6 \%$ to high strain, and $94.9 \%$ reported high social support at work. The average WAI score was 42.4 $(\mathrm{SD}=4.7) ; 20.8 \%$ of participants exhibited early functional ageing (moderate/poor work ability). At the end of follow up $524(49.4 \%)$ participants had job interruption.

Features involved with job interruption were: early functional ageing (OR 1.42; $\mathrm{p}=0.031)$; high strain $(\mathrm{OR}=1.59$; $\mathrm{p}=0.030)$, age $\geq 40$ years $(\mathrm{OR}=0.54 ; \mathrm{p}<0.001)$; job title Assistants/Attendants (OR=1.71; $\mathrm{p}=0.011)$ and Technicians/ Nursing. Assistants/Cleaners $(\mathrm{OR}=1.64 ; \mathrm{p}=0.006)$; employees of all other departments compared to the Clinical Department $(\mathrm{OR}=1.41 ; \mathrm{p}=0.020)$. The model was adjusted by sex.

Discussion Early functional ageing was a risk factor for job interruption in short-to-medium-run in the investigated population. Contrary to expected age was a protective factor. Inadequate work conditions and the psychosocial work environment contributed to the negative outcome.

\section{THE IMPACT OF OCCUPATIONAL HEALTH ON WORK- LIFE EXPECTANCY, A REPRESENTATIVE DANISH STUDY ON THE YEARS 2010-2016}

Jacob Pedersen*. Statistician, Ph.d., the National Research Centre for the Working Environment, Denmark

\subsection{6/oemed-2018-ICOHabstracts. 126}

Introduction To what degree does the occupational health impact the labour market affiliation in terms of work-life expectancy? This question plays a central role in the Danish flexible labour market system with high labour force participation, relatively generous and accessible social benefits, and a low formal employment protection with a high turnover.

Method A survey of 12.429 employed Danes from 2010 is merged with longitudinal register data on social benefits to gain a follow-up period of approximately five years, but no later than the official pension age of 65 years. By using a Multi-state design and modern survival analysis, we estimate the work-life expectancy and divide the results in periods of work, unemployment, and sickness absence. The analyses are done for five occupational health scales divided into good and poor health.

Results For a 40-49 year old non-smoking women, employed in social health type of work and with elementary school education, and without the possibility of voluntary early retirement, one can 
expect 1.1 year decline in work-life expectancy due to poor emotional strain at work. In addition the expected time spend in sickness absence until pension age will increase by 0.9 years, and the expected time spend in unemployment will increase by 0.3 years. Similar results are made for other profiles, with or without the right to early retirement pension scheme.

Discussion Results suggest a significant influence of occupational health on work-life expectancy and work disability among Danish employees. With the new methods utilising the detailed Danish registers one have the ability to estimate work-life expectancy even for small groups, which is often the case when subgrouping levels of occupational health.

\section{LIFESTYLE AND WORK ABILITY IN A NORWEGIAN GENERAL WORKING POPULATION}

${ }^{1,2}$ Marit Müller de Bortoli*, ${ }^{1}$ Inger M Oellingrath, ${ }^{2}$ Martin Veel Svendsen, ${ }^{2}$ Anne Kristin Møller Fell. ${ }^{1}$ Faculty of health- and social sciences, Department of Nursing and Health Sciences, University College of Southeast Norway, Porsgrunn, Norway; ${ }^{2}$ Department of Occupational and Environmental Medicine, Telemark Hospital, Skien, Norway

\subsection{6/oemed-2018-ICOHabstracts.127}

Introduction There has been increasing attention on work ability promotion and prolonging working life in Norway. The aim of this study was to investigate the association between several lifestyle-related risk factors (unhealthy diet, low physical activity, overweight/obesity and smoking) and self-rated work ability.

Methods This study is based on the Telemark study, a crosssectional population study conducted in Telemark county, Norway in 2013. Complete data on lifestyle-related factors and work ability were obtained for 10434 participants aged 1850 years, all engaged in paid work during the past 12 months. The outcome measure was the first single item question of the work ability index (WAI). We used multiple logistic regression analysis to examine the associations with the independent variables: diet, body mass index, physical activity and smoking. We adjusted for age, gender, education and main occupational group and stratified for age groups (18-30, 31-40 and 41-50).

Results Reduced work ability (score <8) was more likely among obese participants (OR 1.5, 95\% CI: 1.3 to 1.7 ), past and current smokers (OR 1.3,95\% CI: 1.1 to 1.5 and $\mathrm{OR}$ 1.4, 95\% CI: 1.2 to 1.6 respectively), inactive individuals (OR 1.4, 95\% CI: 1.3 to 1.6 ), and persons responding to have an unhealthy diet (OR 1.3, 95\% CI: 1.0 to 1.5). Among participants aged 18-30 years, inactivity and smoking were associated with deceased work ability, while among participants aged 41-50 years, all the studied lifestyle-related factors were significantly associated with decreased work ability.

Discussion Lifestyle-related risk factors were found to be associated with reduced work ability in a general working population aged 18-50. The results indicate that workers may benefit from interventions focusing on multiple life style changes. The findings further indicate an increased importance of lifestyle-related behaviours on work ability with age. The results are considered relevant to occupational intervention health programs aimed at prevention of decreased work ability.

\section{SOCIOECONOMIC STATUS, DEMOGRAPHIC AND PERSONAL FACTORS, AND THE EATING BEHAVIOURS OF CIVIL SERVICE EMPLOYEES: A CROSS-SECTIONAL STUDY}

${ }^{1}$ Judith Grant*, ${ }^{1}$ Jonathan Houdmont, ${ }^{2}$ Fehmidah Munir, ${ }^{3}$ Robert Kerr, ${ }^{4}$ Ken Adley. ${ }^{1}$ University of Nottingham, Nottingham, UK; ${ }^{2}$ Loughborough University, Loughborough, UK; ${ }^{3}$ University of UIster, Newtonabbey, UK; ${ }^{4}$ Northern Ireland Civil Service, Belfast, UK

\subsection{6/oemed-2018-ICOHabstracts. 128}

Introduction This study concerns the relationship between socioeconomic status (SES) and eating behaviours in working populations. Much research exists on this topic in a community setting, however more research is needed in a workplace setting to help inform the targeting of interventions to improve employee health.

Methods The study was conducted in 2014 using a single selfreport anonymous questionnaire. The variables of interest in this study were age, gender and number of dependents, three measures of SES (education, income and job type), Body Mass Index (BMI), and diet. Two items, 'how often do you eat past the point of feeling full?' and 'to what extent does the cost of food influence what you buy?' were introduced into the survey in response to a literature review. Hierarchical linear regression analyses were conducted to examine the variance in eating behaviours.

Result A 20\% response rate was achieved with 6206 responses. All three SES variables, in addition to age, number of dependents and BMI accounted for $9 \%$ of the variance in the cost of food influencing purchase behaviours; age, gender and BMI explained $7 \%$ of the variance in eating past the point of feeling full; gender, BMI and all SES variables explained 1\% of the adjusted variance in vegetable consumption; age, gender and BMI explained $2 \%$ of the variance in fruit consumption and age, gender, BMI, education and salary band explained $5 \%$ of the variance in consumption of a healthy well-balanced diet.

Discussion The findings demonstrate the importance of socioeconomic status in eating behaviours and suggest that demographics also play a significant role in influencing employee eating behaviours. This raises interesting questions as to the feasibility of targeting healthy eating interventions at work on the basis age or BMI.

\section{A SNAPSHOT OF 3887 BELGIAN EMPLOYEE'S WORK- ABILITY: A COMPARISON BETWEEN AGE GROUPS}

\footnotetext{
${ }^{1,2}$ Sofie Vandenbroeck* ${ }^{*}{ }^{1}$ Liesbeth Aerts, 1,3 Liesbeth Daenen, Lieve Vandersmissen,

1,2Lode Godderis. 'Knowledge, Information and Research Centre, IDEWE Group (External Service for Prevention and Protection at Work), Leuven, Belgium; '2Environment and Health, Faculty of Medicine, KU Leuven, Leuven, Belgium; ${ }^{3}$ Department of Rehabilitation Sciences and Physiotherapy, Human Physiology and Anatomy (KIMA), Faculty of Physical Education and Physiotherapy, Vrije Universiteit Brussel, Brussels, Belgium
}

\subsection{6/oemed-2018-ICOHabstracts. 129}

Introduction Working life will increase due to a raise of the legal retirement age. Yet, its success will depend on the willingness and ability of workers to remain at work. Work-ability, the backbone of sustainable employability, should therefore continuously be monitored and promoted. Work-ability is determined by health and functional capacities; competences; values, attitudes and 